\title{
The structure of water in soft contact lenses: near infrared spectroscopy and Aquaphotomics study
}

\author{
J. Munćan, ${ }^{a *}$ J. Šakota Rosić, ${ }^{a}$ I. Mileusnić, ${ }^{a}$ V. Matović, ${ }^{a}$ L. Matija ${ }^{a}$ and R. Tsenkova ${ }^{b}$ \\ aNanoLab, Biomedical Engineering, Faculty of Mechanical Engineering, University of Belgrade, Serbia. E-mail: jmuncan@mas.bg.ac.rs \\ ${ }^{b}$ Biomeasurement Technology Laboratory, Faculty of Agriculture, University of Kobe, Japan
}

This research paper reports the results of application of near infrared spectroscopy and Aquaphotomics to the study of the structure of water in hydrogel materials of soft contact lenses. The purpose of this research was to study and describe changes in water structure within hydrogel materials with low, medium and high water content. Near infrared spectroscopy provides a rapid, non-destructive and reagent-free method of characterisation, while the Aquaphotomics approach to the analysis of the spectra of hydrated contact lenses provides identification of different water molecular species within the complex water structure of hydrogels. Our results show the possibilities of applying Aquaphotomics for better description of the states of water in hydrogels, with more information about water than the currently prevailing "three states" model.

\section{Introduction}

Soft contact lenses are made of hydrogels-a class of polymer materials characterised by their ability to absorb and retain a large amount of water within the interstitial space of their cross-linked network, without dissolution. ${ }^{1}$ In conventional hydrogel materials, permeability for oxygen depends entirely on the water content-the higher the water content, the greater amount of oxygen can be transported to the cornea which is avascular and depends solely on oxygen from the atmosphere. 2,3 This makes water of crucial importance for the functionality of soft contact lenses.

The state of water in conventional hydrogels is usually described using the "three-states" water model which distinguishes water in hydrogels as existing in three thermodynamically different classes, usually described as tightly bound, loosely bound and free water, based on the level of interaction with the polymer network ${ }^{3,4}$ (Table 1). The free water fraction is considered particularly important since it determines the transport properties of the material and will consequently affect both oxygen diffusion and dehydration. 3,4

The existence and quantification of these different water fractions in polymers have been determined through the use of differential scanning calorimetry (DSC), thermogravimetric analysis, nuclear magnetic resonance spectroscopy, infrared spectroscopy and chromatography. ${ }^{3}$ There are many issues with the current state of the art techniques and the proposed three-state model of water in hydrogels. For example, the DSC curves of total freezing water in hydrogels (free water and loosely bound water) show very complex

Table 1. Three-state model of water makes distinction between three thermodynamically different classes.

\begin{tabular}{|l|l|l|l|}
\hline Water in hydrogels & Tightly bound water & Loosely bound water & Free water \\
\hline Interaction with polymer & Strong & Intermediate & Negligible \\
\hline Freezable & No (not even at $\left.-100^{\circ} \mathrm{C}\right)$ & Yes (below $\left.{ }^{\circ} \mathrm{C}\right)$ & Yes (at $\left.\mathrm{O}^{\circ} \mathrm{C}\right)$ \\
\hline Also known as & Primary bound & $\begin{array}{l}\text { Secondary bound } \\
\text { Intermediate }\end{array}$ & $\begin{array}{l}\text { Unbound } \\
\text { Bulk }\end{array}$ \\
\hline
\end{tabular}

\section{Correspondence}

J. Munćan (jmuncan@mas.bg.ac.rs)

doi: 10.1255/nir2017.099

Citation: J. Munćan, J. Šakota Rosić, I. Mileusnić, V. Matović, L. Matija and

R. Tsenkova, "The structure of water in soft contact lenses: near infra-

red spectroscopy and Aquaphotomics study", in Proc. $18^{\text {th }}$ Int. Conf. Near

Infrared Spectrosc., Ed by S.B. Engelsen, K.M. Sørensen and F. van den

Berg. IMPublications Open, Chichester, pp. 99-104 (2019). https://doi.

org/10.1255/nir2017.099

\section{(c) 2019 The Authors}

This licence permits you to use, share, copy and redistribute the paper in any medium or any format provided that a full citation to the original paper is given.

ISBN: 978-1-906715-27-4 
and fine structure, completely different from the simple melting behaviour associated with pure water, which indicates that freezing water may encompass a number of intermediate states. ${ }^{5}$ We believe that the best description of the water state in hydrogels could be explained as a continuum of water states between the primary state which is hydrogen bonded to functional groups in the polymer (tightly bound water) and water that is unaffected by its polymeric environment (free water).

What clearly is missing in the current research of water in hydrogels or water-swollen polymer systems in general, is a method sensitive enough to address the described problems and to detect distinct water molecular conformations within this continuum of water states and assign different roles and functionalities to them. Furthermore, the method should not be destructive.

Our intention with this work is to propose application of near infrared (NIR) spectroscopy and Aquaphotomics ${ }^{6}$ as a novel tool, perfectly suited to answer the unresolved questions about the state of water in water-swollen polymer systems. Non-destructive NIR spectroscopy has been widely applied for the last 40 years for evaluating the quality of agricultural and pharmaceutical products, ${ }^{7}$ but in the last decade its use is rapidly expanding to include water structural analyses. ${ }^{6,8}$ The Aquaphotomics approach to analysis of the spectra of water and aqueous systems offers a unique opportunity to describe the complex state of water based on its multidimensional NIR spectra. ${ }^{6}$

The objective of this study is the exploration of the state of water in conventional hydrogel-based soft contact lenses with different water content. It is a continuation of previous studies on the state of water in different hydrogel materials with low and high water content ${ }^{9}$ and on the state of water in worn (spoiled) soft contact lenses. ${ }^{10}$

\section{Materials and method Soft contact lenses}

Three soft contact lens materials were used, all based on the poly-HEMA polymer formulations made by a company Vista Optics, England: VSO38 (pHEMA Filcon I 1, nominal water content $(n w c)=38.4 \%)$, VSO42 (Co-polymer of HEMA \& VP Filcon I 1, nwc = 41.7\%) and VSO50 (Co-polymer of HEMA \& VP Filcon II 1 , nwc $=51.0 \%$ ). The used materials are synthetic hydro- gels based on HEMA (2-Hydroxy-ethyl methacrylate), frequently used monomer in synthesis of hydrogels and combined with VP (Vinyl pyrrolidone)-very hydrophilic monomer, commonly used to increase water content. Contact lenses were lathe-cut with the same parameters: Base curve: $8.60 \mathrm{~mm}$ diameter: $14.20 \mathrm{~mm}$, Sph: +1.00 D (manufacturer Optix DOO, Serbia). Six lenses were fabricated from each material.

\section{Near infrared spectroscopy}

Near infrared spectra of contact lenses were acquired in transmittance mode using a minispectrometer (TG - Cooled NIR-IC9913GC, Hamamatsu, Japan) and transmission fixture TXF-4 (Stellarnet, US) equipped with an annular plastic holder for contact lenses to ensure the fixed, horizontal position of the lenses and to prevent environmental influences during measurements. The transmittance spectra were acquired in the region of 900-1700 nm with a resolution of $7 \mathrm{~nm}$. Before each spectral acquisition a spectrum of the empty holder was used as a reference. All measurements were done when lenses were in a fully swollen state. Excess solution was removed by quick dabbing of lenses with Kim wipe tissue papers immediately before placing the lens in the holder. Five consecutive spectral replicas were acquired per each lens. Experiments were repeated several times, giving in total 270 spectra of lenses for the analysis.

\section{Data analysis}

Data analysis of absorbance spectra $\left(\log T^{-1}\right)$ was carried out using Pirouette ver. 4.5 (Infometrix Inc.) and R software with the Aquap2 package-Multivariate Data Analysis Tools for R with Aquaphotomics methods. ${ }^{11}$

The spectral range was trimmed to $1300-1600 \mathrm{~nm}$ before the analysis. To minimise the differences between spectra caused by baseline shift and noise, prior to the analysis spectral data were mean-centred, transformed using standard normal variate (SNV) transformation ${ }^{12}$ and smoothed using the Savitzky-Golay algorithm ${ }^{13}$ (19 pts). The spectral data were explored using Principal Component Analysis ${ }^{14}$ and spectra identified as outliers according to Mahalanobis distance were removed from the datasets. The model was validated using step-validation (leave-5-out).

The water absorbance values at specific water matrix coordinates (WAMACs) defines the water spectral 
pattern-WASP. ${ }^{6}$ The visualisation of WASP for different contact lenses materials was done using a spider web plot called aquagram ${ }^{8}$ which shows normalised absorbance values at chosen wavelengths from the WAMACs ranges. The classic mode of Aquagram with confidence intervals $^{15}$ calculated for selected wavelengths was chosen to display the WASP of contact lenses with different water content and to find statistically significant differences in WASP of different materials. The respective confidence interval with 95\% upper and lower limits are calculated using Bootstrap method for data validation and uncertainty estimation. ${ }^{16}$ All aquagrams were calculated based on SNV-treated and smoothed spectral data.

\section{Results and discussion}

The raw absorbance spectra of soft contact lenses are presented in Figure 1. Since water is an integral part of the hydrogel materials, the spectra are dominated by water spectral features in this NIR region. The raw spectra show significant baseline offset caused probably by the different central thickness of the lenses, which, even though the nominal thickness is the same for all lenses, may vary due to immediate dehydration during handling and measurements. To correct these effects standard normal variate transformation was used, and spectra were smoothed to remove the noise.

To enquire about the differences in the water spectral pattern of soft contact lenses materials, twelve wave-

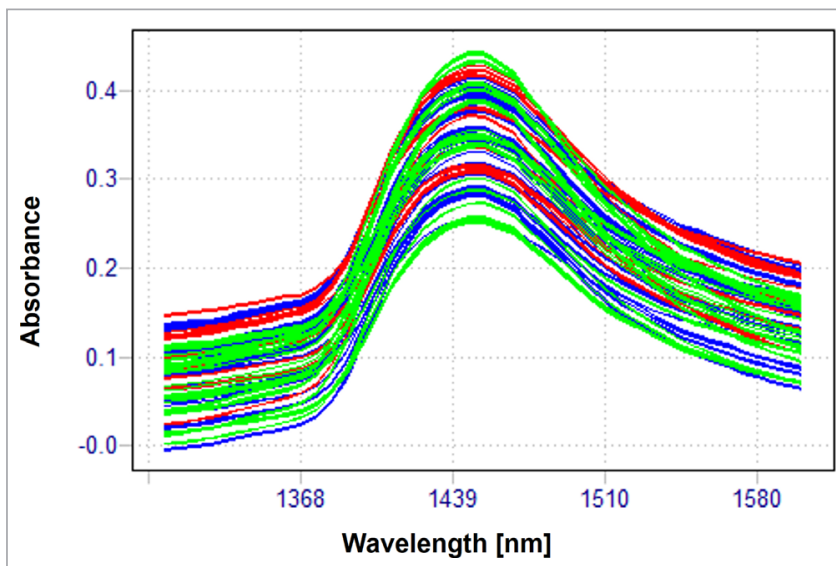

Figure 1. Raw absorbance spectra of soft contact lenses: pHEMA polymer - $38 \%$ water content (VSO38 - blue lines), HEMA\&VP copolymer - $42 \%$ water content (VSO42 - red lines) and HEMA\&VP copolymer - 50\% water content (VSO50 - green lines).

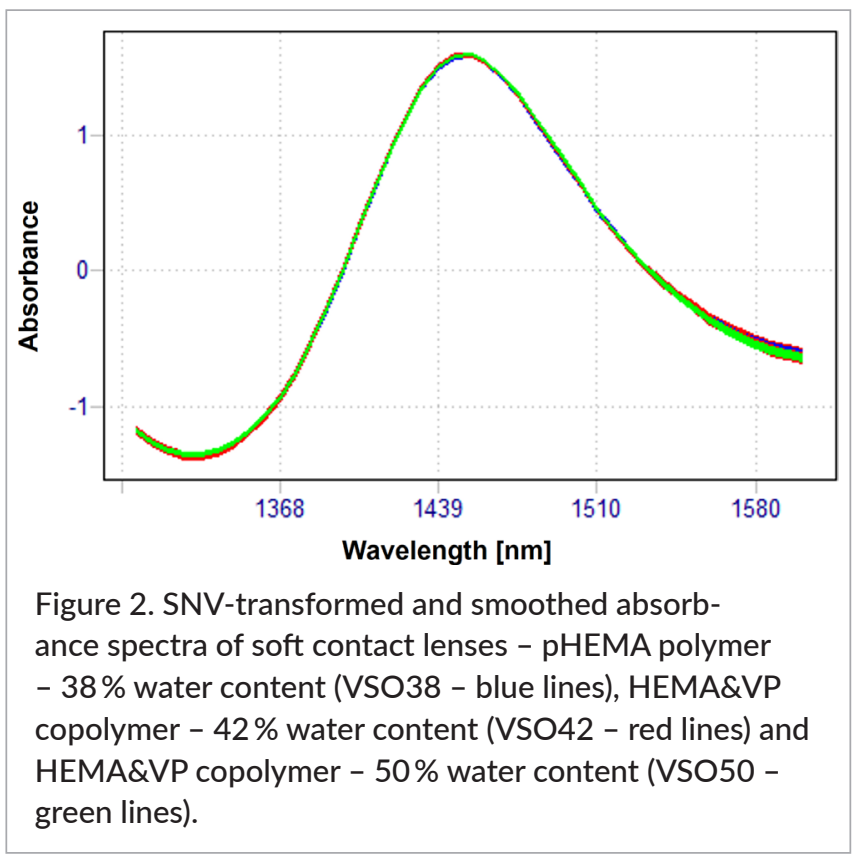

lengths were selected from defined water matrix coordinates (WAMACs) ranges. All WAMACs correspond to specific water absorbance bands of different water molecular conformations. To visualise the WASP of different soft contact lenses materials, aquagrams with confidence intervals were used, which display bootstrap estimates of averaged normalised absorbance and low and high 95\% confidence limits for each material (Figure 2). The confidence intervals allow identification of only statistically significant differences in absorbance at different WAMACs of the material WASPs (Table 2).

The same major structural component (HEMA) was chosen for all materials to facilitate easier identification of water absorbance bands of specific water molecular conformations which could be associated with defined classes of free, loosely bound and tightly bound water. In hydrogel materials derived from the same polymer system-increasing water content will not change the amount of tightly bound water. ${ }^{3}$ Comparison of the water spectral pattern of different materials presented in aquagrams show that only for absorbance at C12 water matrix coordinate, there are no statistically significant differences between materials (Table 2, C12: $1517 \mathrm{~nm}$ ). This finding leads to the conclusion that this band is the absorbance band of tightly bound water in polymer, also called non-freezing bound water which makes a complex with polymer chains through hydrogen bonds, 5,17 and is lost only when the polymer network is damaged. ${ }^{10}$ 

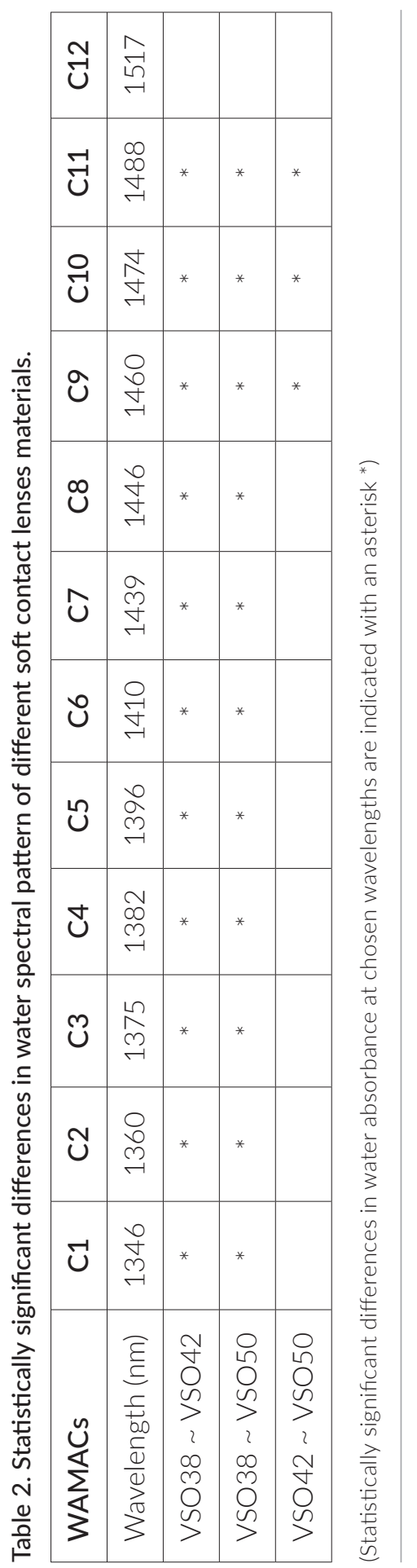

Introduction of vinyl pyrrolidone to pHEMA in VSO42 and VSO5O materials leads to significantly increased absorbance at all other WAMACs, which suggests that these bands represent bands of loosely bound and free water. Analysis of the statistically significant differences in absorbance of VSO42 and VSO5O materials shows that they differ significantly in absorbance at C9, C10

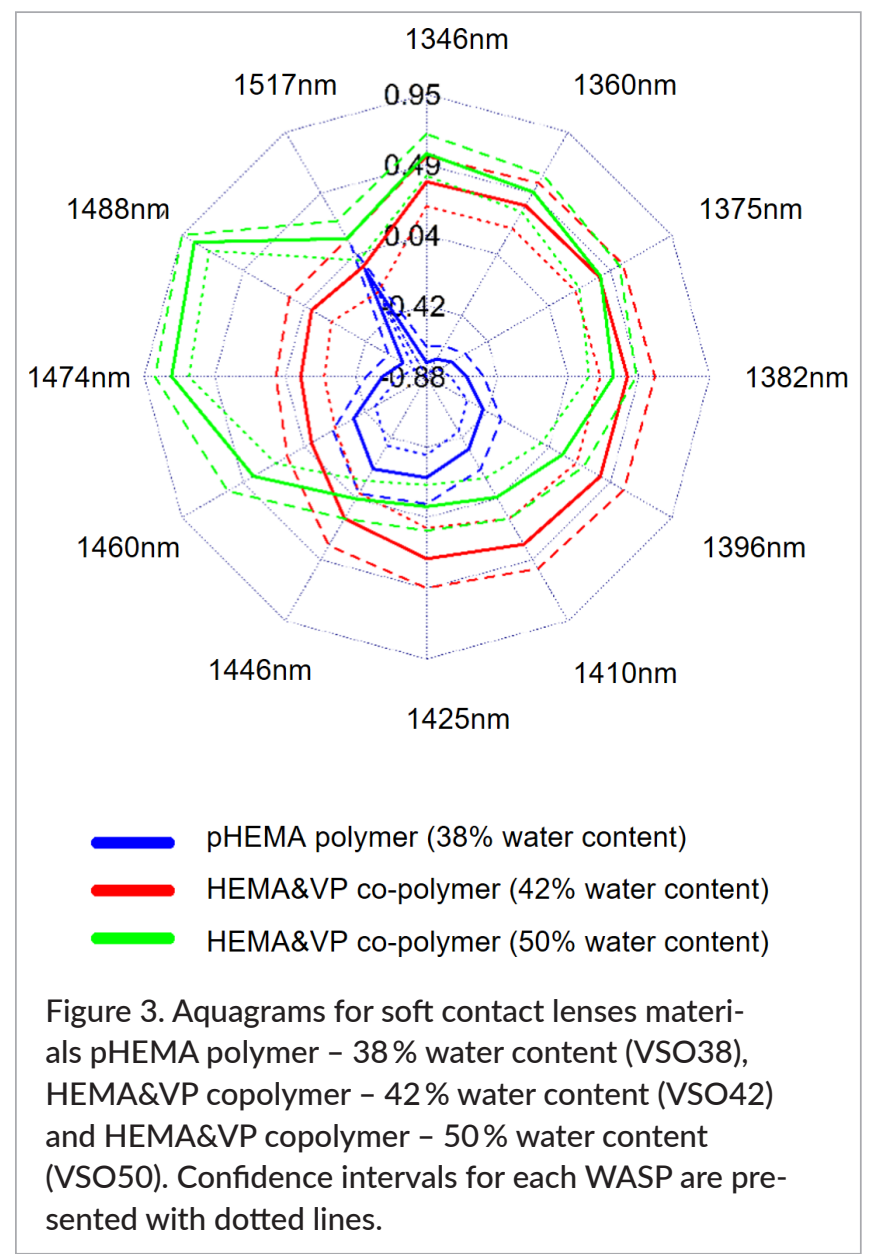

and C11 WAMACs (Table 2, $1460 \mathrm{~nm}, 1474 \mathrm{~nm}$ and $1488 \mathrm{~nm}$ ) which are the bands of water molecules with 2, 3 and 4 hydrogen bonds, ${ }^{6}$ respectively. Loosely bound water is, of all water fractions the least defined, but what is known is that it encompasses water species with higher hydrogen-bonding energies than pure water, ${ }^{3}$ i.e. water molecules forming hydrogen bonds with other water molecules, a necessary condition for water to be able to freeze-which is why this water is also known as a freezable bound water. ${ }^{3}$ In a recent study, also, for pHEMA hydrogel co-polymers with vinyl pyrrolidone, it was found that with increased water content, the amount of free water stays almost constant. ${ }^{17}$ On the basis of this information, we can tentatively conclude that C9C11 water matrix coordinates ${ }^{6}$ are absorbance bands of specific water molecular conformations belonging to the loosely bound water fraction. The free water fraction, in that case, is represented by water molecules absorbing at C1-C6 WAMACs, ${ }^{6}$ which correspond to free water molecules, water molecules with free $\mathrm{OH}$ bond and water molecules in hydration shells. 


\section{Conclusion}

The results presented in this paper show that near infrared spectroscopy allows characterisation of conventional hydrogel-based soft contact lenses, in the conditions similar to physiological (hydrated contact lenses). It allows longer path lengths because near infrared light passes through the entire contact lens in a completely nondestructive manner. The application of Aquaphotomics approach to the analysis of near infrared spectra of contact lenses provides detailed description of the water species existing in the polymer network and allows qualitative comparison of water in different hydrogel materials. It goes beyond the "three-state" model of water revealing different water species within the classes of loosely bound and free water.

\section{Acknowledgement}

This study was supported by the Ministry of Science and Education of Republic of Serbia (Project III45009).

\section{References}

1. K. Dušek and J. Šomvársky, "Modelling of ring-free crosslinking chain (co) polymerization", Polym. Int. 44, 225-236 (1997). https://doi.org/10.1002/(SICI)10970126(199711)44:3<225::AID-PI870>3.0.CO;2-C

2. G. Andrasko, "Hydrogel dehydration in various environments", Int. Contact Lens Clin. 10, 22-28 (1983). https://doi.org/10.1111/j.1475-1313.1988. tb01055.x

3. I. Tranoudis and N. Efron, "Water properties of soft contact lens materials", Cont Lens Anterior Eye 27, 193-208 (2004). https://doi.org/10.1016/j. clae.2004.08.003

4. K. Krysztofiak and A. Szyczewski, "Study of dehydration and water states in new and worn soft contact lens materials", Opt. Appl. 44, 237-250 (2014). https://doi.org/10.5277/oa140206

5. D.G. Pedley and B.J. Tighe, "Water binding properties of hydrogel polymers for reverse osmosis and related applications", Polym. Int. 11, 130-136 (1979). https://doi.org/10.1002/pi.4980110306

6. R. Tsenkova, "Introduction: Aquaphotomics: dynamic spectroscopy of aqueous and biological systems describes peculiarities of water", J. Near Infrared Spectrosc. 17, 303-313 (2010). https://doi. org/10.1255/jnirs.869

7. P. Williams and K. Norris, Near-Infrared Technology in the Agricultural and Food Industries. American Association of Cereal Chemists, Inc., St Paul, Minnesota, USA (1987).

8. R. Tsenkova, "Aquaphotomics: water in the biological and aqueous world scrutinised with invisible light", Spectrosc. Europe 22(6), 6-10 (2010). https://www. spectroscopyeurope.com/article/aquaphotomicswater-biological-and-aqueous-world-scrutinisedinvisible-light

9. J. Munćan, I. Mileusnić, J. Šakota Rosić, A. VasićMilovanović and L. Matija, "Water properties of soft contact lenses: a comparative nearinfrared study of two hydrogel materials", Int. J. Polym. Sci. 2016, 3737916 (2016). https://doi. org/10.1155/2016/3737916

10. J. Šakota Rosić, J. Munćan, I. Mileusnić, B. Kosić and L. Matija, "Detection of protein deposits using NIR spectroscopy", Soft Materials 14, 264-271 (2016). https://doi.org/10.1080/1539445X.2016.1198377

11. B. Pollner and Z. Kovacs, Multivariate Data Analysis Tools for R including Aquaphotomics Methods (2016).

12. R. Barnes, M.S. Dhanoa and S.J. Lister, "Standard normal variate transformation and de-trending of near-infrared diffuse reflectance spectra", Appl. Spectrosc. 43, $772-777$ (1989). https://doi. org/10.1366/0003702894202201

13. A. Savitzky and M.J. Golay, "Smoothing and differentiation of data by simplified least squares procedures", Anal. Chem. 36, 1627-1639 (1964). https:// doi.org/10.1021/ac60214a047

14.I.A. Cowe and J.W. McNicol, "The use of principal components in the analysis of near-infrared spectra", Appl. Spectrosc. 39, 257-266 (1985). https://doi. org/10.1366/0003702854248944

15. B. Pollner and Z. Kovacs, Multivariate Data Analysis Tools for R including Aquaphotomics Methods, aquap2 (2016).

16.A.C. Davison and D.V. Hinkley, Bootstrap Methods and their Applications. Cambridge Series in Statistical and Probabilistic Mathematics, Cambridge University Press, Vol. 32, pp. 10013-12473 (1997).

17.E. Seo, S. Kumar, J. Lee, J. Jang, J.H. Park, M.C. Chang, I. Kwon, J-S. Lee and Y-i. Huh, "Modified hydrogels based on poly (2-hydroxyethyl 
methacrylate)(pHEMA) with higher surface wettability and mechanical properties", Macromolecular Res. 25, 704-711 (2017). https://doi.org/10.1007/ s13233-017-5068-y 